\title{
Prediction of fragmentation of kidney stones: A statistical approach from NCCT images
}

\author{
Krishna Moorthy, MD; Meenakshy Krishnan, $M D^{2}$ \\ 'Lourdes Hospital, Kochi, India; '2Department of Electrical and Electronics, Government Engineering College, Thrissur, S. India
}

Cite as: Can Urol Assoc J 2016;10(7-8):E237-40. http://dx.doi.org/10.5489/cuai.3674 Published online July 12, 2016

\section{Abstract}

Introduction: We sought to develop a system to predict the fragmentation of stones using non-contrast computed tomography (NCCT) image analysis of patients with renal stone disease.

Methods: The features corresponding to first order statistical (FOS) method were extracted from the region of interest in the NCCT scan image of patients undergoing extracorporeal shockwave lithotripsy (ESWL) treatment and the breakability was predicted using neural network.

Results: When mean was considered as the feature, the results indicated that the model developed for prediction had sensitivity of $80.7 \%$ in true positive (TP) cases. The percent accuracy in identifying correctly the TP and true negative (TN) cases was $90 \%$. $\mathrm{TN}$ cases were identified with a specificity of $98.4 \%$.

Conclusions: Application of statistical methods and training the neural network system will enable accurate prediction of the fragmentation and outcome of ESWL treatment.

\section{Introduction}

Extracorporeal shockwave lithotripsy (ESWL) has been widely accepted as the treatment of choice for renal stones less than $2 \mathrm{~cm}$, with variable success rates of 60-99\% across the globe. The success rate of this procedure could be influenced by stone factors (stone size, location, composition, degree of obstruction), clinical factors (comorbidities, such as concomitant infection, solitary kidney, abnormal ureteral anatomy), and technical factors (available equipment, source of energy). ${ }^{1}$ Several other factors, such as body mass index (BMI) and stone to stone distance (SSD) also influence the stone clearance and success rate. ${ }^{2}$ In addition, the attenuation values of calculi measured on computed tomography (CT) in Hounsfield units (HU) has also been studied to determine the success rate of ESWL or stone fragmentation. ${ }^{3}$
Since the1990s, non-contrast enhanced computed tomography (NCCT) has become the radiographic modality of choice in the diagnosis of urinary stone disease, with sensitivity of $96 \%$, specificity of $99 \%$, and accuracy of $98 \% .{ }^{4,5}$ While Joseph et $\mathrm{al}^{6}$ observed that the best results of ESWL were obtained with stone densities less than 1000 HU measured by NCCT, Gupta et $\mathrm{al}^{7}$ recommended ESWL as the first choice for fragmentation of stones with density less than $750 \mathrm{HU}$.

It is well-known that most urinary stones are made up of a mixture of different components, predominantly calcium, oxalate, phosphate, and uric acid in varying quantities, which predictably can alter the composition and density of stones in the various regions. The majority of studies comparing the stone fragility to the stone density had considered that the stones were made of single material and had uniform density in all regions. Therefore, the highest or mean attenuation values in a few areas in the region of study were calculated and compared to the success rate of ESWL. This could probably alter the comparative results of stone fragmentability vs. stone density measured in NCCT. Moreover Zarse et $\mathrm{al}^{8}$ had also reported that NCCT might not be able to identify stone composition based on densities measured in HU, especially when 3-5 mm slices were taken as soft tissue windows to increase stone brightness, due to alterations in the HU values.

Therefore, it is imperative to develop a foolproof method to identify the density of renal stones in vivo and correlate this with the success rate of ESWL. This method could further be extended to predict the fragility of stones before subjecting them to ESWL treatment, so as to improve the success rates of this non-invasive modality of treatment.

\section{Methods}

For this study, a total of 120 patients aged 20-60 years with renal stones of size 1-2 cm were selected for ESWL treatment using Direx Lithotripter during a period of 12 months. 
The patients were subjected to NCCT images prior to the procedure. Each patient was given 2500 shockwaves at a power setting of 60-75 KV and intensity setting of $7.2 \mathrm{~mA}$ under sedoanalgesia. The patients were followed up one month after the procedure and a repeat NCCT was taken. Fragility and success of ESWL was defined as the complete absence of stones or presence of residual stone fragments less than $3 \mathrm{~mm}$ size. Patients with larger stones (more than 2 $\mathrm{cm}$ ), multiple stones, obstructed stones with severe grades of hydronephrosis, renal failure, and obese patients with body weight more than $100 \mathrm{~kg}$ were excluded from the study.

Out of the 120 patients studied, the images of 80 patients were selected for training the neural network and the images of a subsequent 40 patients were used for test prediction of fragility. The sample size was calculated as per statistical norms using software.

From the segmented image obtained from NCCT, the matrix corresponding to the region of interest (ROI) was first obtained. The matrix gave the grey level values in the range of $0-255$ corresponding to the attenuation values obtained from NCCT. Each grey level value of the matrix corresponding to the image was termed as pixel. This matrix formed the input to the feature extraction module. The first order statistical features (FOS), such as mean, variance, skewness, and kurtosis, were calculated from the grey level matrix using the equations given below, using the Matlab software.

$$
\begin{aligned}
& \operatorname{Mean}(\mu)=\sum_{i=1}^{N_{g}} x_{i} n_{i} / n \\
& \operatorname{Variance}\left(\sigma^{2}\right)=\sum_{i=1}^{N_{g}}\left(x_{i}-\mu\right)^{2} n_{i} / n \\
& \operatorname{Skewness}(\mu 3)=\frac{1}{\sigma^{3}} \sum_{i=1}^{N_{g}}\left(x_{i}-\mu\right)^{3} n_{i} / n \\
& \operatorname{Kurtosis}(\mu 4)=\frac{1}{\sigma^{4}} \sum_{i=1}^{N_{g}}\left(x_{i}-\mu\right)^{4} n_{i} / n
\end{aligned}
$$

$N_{g}$ : number of grey levels; $x_{i}: i^{\text {th }}$ grey level; $n_{i}$ : number of pixels with $x_{i}$ grey levels; $n$ : total number of pixels.

Mean represented the average value. Variance measured how similar the data were within a region. Skewness was a measure of asymmetry of the data around the sample mean.
If skewness was negative, the data was spread more to the left of the mean than to the right. If skewness was positive, the data was spread out more to the right. Kurtosis was the degree of peakedness of the distribution, defined as a normalized form of the fourth central moment of distribution.

Artificial neural network (ANN) was used to classify the data. ${ }^{9}$ ANN was trained to associate outputs with input patterns. Training required the pairing of each input vector with a target vector representing the desire output. A network needed to be trained over a number of training pairs until it reached a user-defined performance level.

The results of classification were expressed in terms of true positive (TP), true negative (TN), false positive (FP), and false negative (FN). A TP meant that the image of a fragmentable stone was classified as fragmentable and TN a non-fragmentable image was classified as non-fragmentable. A ROI corresponding to a fragmentable stone classified as non-fragmentable was FP and a non-fragmentable identified as fragmentable was FN. From these measures, the sensitivity, specificity, and accuracy of the method were also evaluated. ${ }^{10}$

$$
\begin{aligned}
& \text { Sensitivity }=\frac{T P}{T P+F N} \\
& \text { Specificity }=\frac{T N}{T N+F P} \\
& \text { Accuracy }=\frac{T P+T N}{T P+T N+F P+F N}
\end{aligned}
$$

Accuracy measured the ability of the classifier to produce correct diagnosis. Sensitivity measured the ability of the model to correctly identify the occurrence of a target class. Specificity measured the ability of the model to separate the target class. ${ }^{11} \mathrm{~A}$ feature wrapper approach was used for feature selection. ${ }^{12,13}$

\section{Results}

Out of the 120 patients included in the study, 93 patients were male and the rest were female; ratio of left-side to right-sided stone was 68:52. Eighty-six (86) patients were below the age of 40 years.

Of the 120 images selected for the study, 47 were fragmentable and 73 were non-fragmentable, as identified by followup NCCT. The training of the neural network was performed using features obtained from 80 images, out of which 39 were fragmentable and 41 non-fragmentable. The remaining images were used for testing. The training and classification were performed using individual features and combination of features. The results obtained for individ- 
ual features, combination of two features, three features, and four features when classified using the trained ANN are shown in Table 1.

When mean was considered as the feature, the results indicated that the model developed for prediction had sensitivity of $80.7 \%$ in TP cases. The percent accuracy in identifying correctly the TP and TN cases was $90 \%$. TN cases were identified with a specificity of $98.4 \%$.

With variance as the feature, the sensitivity was only $57.5 \%$, and accuracy $70.8 \%$, while specificity was $97.5 \%$. Skewness as the feature yielded $64.7 \%$ sensitivity, $77.5 \%$ accuracy, and $94.2 \%$ specificity. Kurtosis resulted sensitivity, accuracy and specificity values of $66.7 \%, 75.8 \%$, and $87.3 \%$, respectively. When various combination of features were considered, the sensitivity and accuracy values showed deterioration, although there was a marginal improvement in the specificity. The combinations of mean/skewness and mean/kurtosis gave the same or similar values for sensitivity, specificity, and accuracy, as obtained for mean as a single feature. The other combinations of features, such as variance/skewness, variance/kurtosis, skewness/kurtosis, variance/skewness/kurtosis, showed a decrease in values for sensitivity, specificity, and accuracy.

\section{Discussion}

Though ESWL is a safe and effective method of treatment of renal stones that causes minimal complication, unfavourable composition of stone has been regarded as a major factor for failure of this mode of treatment. Therefore, it is important that some information regarding composition of stone must be known before identifying a patient for ESWL treatment. This information can be obtained from a report of stone analysis, which is only possible in patients with recurrent stone disease. Some studies done previously predicted the success of ESWL from analyzing the ultrasound and plain radiographic images of renal stones prior to the treatment, with varying success rates of $39-40 \% .^{14,15}$

Today, diagnosis of urinary stones can be done by more effective methods, such as helical NCCT, with sensitivity, specificity, and accuracy over $95 \%$. Chua et $\mathrm{al}^{16}$ determined the diagnostic utility of CT scout film with an optimal noncontrast helical CT scan HU in predicting the appearance of urinary calculus in the plain kidney-ureter-urinary bladder (KUB) radiograph. It has been reported that each class of mineral in the stone gives a different attenuation value in NCCT. Urate has been reported to have attenuation values of $513 \pm 292 \mathrm{HU}$, while calcium oxalate values are 1684 $\pm 290 \mathrm{HU}^{17}$

Various studies have been done to ascertain the role of HU unit values in predicting the success of ESWL. ${ }^{6,7,14-18}$ Ouzid et al ${ }^{19}$ reported that a stone density of $970 \mathrm{HU}$ represented the most sensitive (100\%) and specific (81\%) point on prediction of fragmentability, with stone-free rates for the group with $<970 \mathrm{HU}$ being $96 \%$ vs. $38 \%$ for the group with $\mathrm{HU}>970 \mathrm{HU}$. The success rate of ESWL was reported to be $94-100 \%, 85.7 \%$, and $54.5 \%$ in the groups with $\mathrm{HU}$ $<500,500-1000$, and $>1000$ respectively. ${ }^{17}$ Weld et al, ${ }^{20}$ however, noted that stone size and intrarenal location of stone also predicted the ESWL fragmentation rates. Pareek et $\mathrm{al}^{2-4}$ reported that $\mathrm{BMI}, \mathrm{SSD}$, and $\mathrm{HU}$ significantly predict the outcome of ESWL fragmentation; they concluded that SSD of more than $10 \mathrm{~cm}$ predicted treatment failure. Cariroglu et $\mathrm{a}^{21}$ found significant correlation between stone size and $\mathrm{HU}$ and also between stone size and the number of shockwaves required for fragmentation. The influence of internal stone structure on the fragmentation behaviour of urinary calculi had also been studied by Pittomul et $\mathrm{a}^{22}$ Similarly, in vitro studies done by Marchinena et $\mathrm{a}^{23}$ also proved that there was a statistically significant relationship between the attenuation values and fragility.

Thus, it is evident from various studies that the attenuation values of stones measured in $\mathrm{HU}$ was a useful method to ascertain the fragility of stones by ESWL. However, the majority of urinary stones often contain a combination of one or more of minerals and, therefore, the attenuation values assessed by NCCT could widely vary in different regions of the stone. The relevance of considering a single

Table 1. Results of classification of stone features

\begin{tabular}{lccccccccccccccc}
\hline \multicolumn{10}{c}{ Selected feature } \\
\hline & $\mu$ & $\sigma^{2}$ & $\mu_{3}$ & $\mu_{4}$ & $\mu+\sigma^{2}$ & $\mu+\mu_{3}$ & $\mu+\mu_{4}$ & $\sigma^{2}+\mu_{3}$ & $\sigma^{2}+\mu_{4}$ & $\mu_{3}+\mu_{4}$ & $\begin{array}{c}\mu+\mu_{4}+ \\
\sigma^{2}\end{array}$ & $\begin{array}{c}\mu+ \\
\mu_{4}+\mu_{3}\end{array}$ & $\begin{array}{c}\mu+\sigma^{2}+ \\
\mu_{3}\end{array}$ & $\begin{array}{c}\sigma^{2}+\mu_{3}+ \\
\mu_{4}\end{array}$ & $\begin{array}{c}\mu+\sigma^{2} \\
+\mu_{3}+\mu_{4}\end{array}$ \\
\hline TP & $\mathbf{4 6}$ & 46 & 44 & 36 & 47 & 46 & 47 & 46 & 46 & 43 & 47 & 47 & 47 & 46 & 47 \\
TN & $\mathbf{6 2}$ & 39 & 49 & 55 & 42 & 62 & 61 & 21 & 43 & 59 & 42 & 59 & 42 & 43 & 42 \\
FP & $\mathbf{1}$ & 1 & 3 & 11 & 0 & 1 & 0 & 1 & 1 & 4 & 0 & 0 & 0 & 1 & 0 \\
FN & $\mathbf{1 1}$ & 34 & 24 & 18 & 31 & 11 & 12 & 52 & 30 & 14 & 31 & 14 & 31 & 30 & 31 \\
Total & $\mathbf{1 2 0}$ & 120 & 120 & 120 & 120 & 120 & 120 & 120 & 120 & 120 & 120 & 120 & 120 & 120 & 120 \\
Sensitivity & $\mathbf{8 0 . 7}$ & 57.5 & 64.7 & 66.7 & 60.3 & 80.7 & 79.7 & 46.9 & 60.5 & 75.4 & 60.3 & 77 & 60.3 & 60.5 & 60.3 \\
Specificity & $\mathbf{9 8 . 4}$ & 97.5 & 94.2 & 87.3 & 100 & 98.4 & 100 & 95.5 & 97.7 & 93.7 & 100 & 100 & 100 & 97.7 & 100 \\
Accuracy & $\mathbf{9 0 . 0}$ & 70.8 & 77.5 & 75.8 & 74.2 & 90.0 & 90.0 & 55.8 & 74.2 & 85.0 & 74.2 & 88.3 & 74.2 & 74.2 & 74.2 \\
\hline TP: true positive; TN: true negative; FP: false positive; FN: false negative. & & & & & & & & & &
\end{tabular}


HU value or mean value obtained from a few areas of the stone is questionable. Our statistical method helps to avoid the shortcomings of the existing system, since the mean is taken from the whole matrix corresponding to the ROI of the stone image.

NCCT clearly helped to get cross-sectional images from the hard, central part of the stone. The initial training of the neural network enabled standardization of the procedure for accurate prediction of stone fragmentation. Since the specificity obtained is high, the chance of classifying a nonfragmentable as fragmentable with the model created is only $2.6 \%$. Even though the values for sensitivity, specificity, and accuracy were the same for classification performed with mean alone, mean/skewness together and mean/kurtosis together, mean alone gave the best results. The subsequent use of neural network yielded results with a high degree of predictability. Valid research methods have been employed for interpretation in this study.

The main limitations of the study were that the authors have not evaluated the breakability of larger stones more than $2 \mathrm{~cm}$ size (which were likely to have a significant difference in density in various areas of the same stone) and the fragmentability of stones with varying intensities of shockwaves applied. The clinical application of this method in patients with multiple stones (of different densities) is also doubtful.

\section{Conclusion}

Measurement of attenuation values of stones using NCCT to determine stone fragmentability before ESWL treatment has various drawbacks. However, application of statistical methods and training the neural network system will help to accurately predict the fragmentation and outcome of ESWL treatment in these patients. Since this method uses the imaging technique and a neural network, an automated system could be developed in future to be attached to the $\mathrm{CT}$ machine for prediction of fragmentation of stones.

Competing interests: The authors declare no competing personal or financial interests.

This paper has been peer-reviewed.

\section{References}

1. Lingaman JE, Matlaga BR. Surgical management of upper tract calculi. In: Campbell Walsh Urology Wein AJ, Kavouisi LR, Novick AC, Patrick AW, Peters CA (Eds). 10th Edition, Saunders, Philadelphia. 2010; 1375-77.
2. Pareek $G$, Armenakar NA, Panagopoulos $G$, et al. Extracorporeal shockwave lithotripsy success based on body mass index and Hounsfield units. Urology 2005;65 33-6. http://dx.doi.org/10.1016/i. urology.2004.08.004

3. Pareek G, Armenakar NA, Fracchia JA. Hounsfield units in a computerized tomography predict stone free rates after extracorporeal shockwave lithotripsy. J Urol 2003;169:1679-81. http://dx.doi. org/10.1097/01.ju.0000055608.92069.3a

4. Pareek G, Hedican SP, Lee FT, et al. Shockwave lithotripsy success determined by skin to stone distance on computerized tomography. Urology 2005;66:941-4. http://dx.doi.org/10.1016/i.urology.2005.05.011

5. Dalrymple NC, Verga M, Anderson KR, et al. The value of unenhanced helical computerized tomography in the management of acute flank pain. J Urol 1998;159:735-40. http://dx.doi.org/10.1016/ S0022-5347(01)63714-5

6. Joseph P, Mandal AK, Singh SK, et al. Computerized tomography attenuation values of renal calculus: Can it predict successful fragmentation of the calculus by extracorporeal shockwave lithotripsy? A preliminary study. J Urol 2002; 167:1968-71. http://dx.doi.org/10.1016/S0022-5347(05)65064-1

7. Gupta NP, Ansari MS, Kesarvani P, et al. Role of computed tomography with no contrast medium enhancement in predicting the outcome of extracorporeal shockwave lithotripsy for urinary calculi. BJU Int 2005; 95:1285-8. http://dx.doi.org/10.1111/i.1464-410X.2005.05520.x

8. Zarse CA, Hameed TA, Jackson ME, et al. Non-destructive analyssis of urinary calculi using micro computerized tomography. BMC Urol 1994;4:12-25.

9. Fausett L. In: Fundamentals of Neural Networks Architectures, Algorithms, and Applications 2nd Edition, Pearson Education. 2007; 19-56.

10. Wei Q. and Hu Y. A study on using texture analysis methods for identifying lobar fissure regions in isotropic CT images. In: Proceedings of the 31st Annual International Conference of the IEEE, EMBS, USA. 2009; 3537-40. http://dx.doi.org/10.1109/iembs.2009.5333083

11. Loo CK and Rao MVC. Accurate and reliable diagnosis and classification using probabilistic ensemble simplified fuzzy ARTMAP. IEEE Trans Knowl Data Eng 2005; 17: 1589-93. http://dx.doi.org/10.1109/ TKDE.2005.173

12. Kohavi R. Wrappers for feature subset selection export. Artificial Intelligence 1997;97:273-324. http:// dx.doi.org/10.1016/S0004-3702(97)00043-X

13. Roth FP. Bringing out the best features of expression data. Genome Research (Insight/Outlook) 2001;11: 1801-02.

14. Dretler SP, Polycoff G. Calcium oxalate stone morphology: Fine-tuning our therapeutic distinctions. J Urol 1996;155:826-33. http://dx.doi.org/10.1016/S0022-5347(01)66319-5

15. Ramakumar S, Patterson DE, LeRoy AJ, et al. Prediction of stone composition from plain radiographs: A prospective study. J Endourol 1999;13:397-401.

16. Chua ME, Gomez OR, Sapno LD, et al. Use of computed tomography scout film and Hounsfield unit of computed tomography scan in predicting the radio-opacity of urinary calculi in plain kidney, ureter, and bladder radiographs. Urol Ann 2014;6: 218-33. http://dx.doi.org/10.4103/0974-7796.134270

17. Ringden I, Tisehus HG. Composition and clinically determined hardness of urinary stones. Scan I Urol Nephrol 2007;41:316-25. http://dx.doi.org/10.1080/00365590601154551

18. Tarawneh E, Awad Z, Hani A, et al. Factors affecting urinary calculi treatment for extracorporeal shockwave lithotripsy. Saud J Kid Dis Transpl 2010;21:660-5.

19. Ouzaid I, Al-qahtani S, Dominique S, et al. A 970 Hounsfield units (HU) threshold of kidney stone density on non-contrast computed tomography (NCCT) improves patients' selection for extracorporeal shockwave lithotripsy (ESWL): Evidence from a prospective study. BJU Int 2012;110:438-2. http:// dx.doi.org/10.1111/i.1464-410X.2012.10964.x

20. Weld KJ, Montiglio C, Morris MS, et al. Shockwave lithotripsy success for renal stones based on patient and stone computed tomography characteristics. Urology 2007;70:1043-6. http://dx.doi.org/10.1016/i. urology.2007.07.074

21. Cakiroghu B, Eyyupoglu SE, Tas T, et al. Are Hounsfield densities of ureteral stones a predictive factor for the effectiveness of extracorporeal shockwave lithotripsy. Int I Clin Exp Med 2014;7:1276-83.

22. Pittomvils $G$, Vandeursen $H$, Wevers $M$, et al. The influence of internal stone structure upon the fracture behaviour of urinary calculi. Ultrasound Med Biol 1994;20:803-10. http://dx.doi.org/10.1016/03015629(94)90037-X

23. Marchiena GP, Peres BN, Liyo J, et al. CT scan as a predictor of composition and fragility of urinary lithiasis treated with extracorporeal shockwave lithotripsy in vitro. Lithiasis Arch Esp Uro 2009;62:215-21.

Correspondence: Dr. Krishna Moorthy, Lourdes Hospital, Kochi, India; dr.moorthy65@gmail.com 